\section{International Scientific Journal Theoretical \& Applied Science}

p-ISSN: 2308-4944 (print) e-ISSN: 2409-0085 (online)

Year: $2014 \quad$ Issue: $10 \quad$ Volume: 18

Published: $30.10 .2014 \quad$ http://www.T-Science.org

SECTION 2 Applied Mathematics. Mathematical modeling.

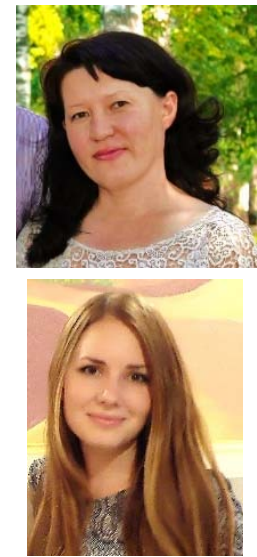

Guzel Rafkatovna Galiaskarova

candidate of physic-mathematical sciences, docent, Sterlitamak branch of the Bashkir

State University, Russia gguselia@mail.ru

Olga Vladimirovna Kulinich student

Sterlitamak branch of the Bashkir

State University, Russia

olkokulinich@yandex.ru

\title{
DYNAMIC MODEL OF OPTIMAL PLACEMENT OF INDUSTRIAL ENTERPRISES
}

Abstract: The main goal is to design a dynamic model of optimal allocation of bakery businesses in several areas of the city. This model provides an efficient distribution construction of new plants in several areas of the city or cities by reducing the cos of their construction.

Key words: dynamic programming, the optimal allocation of enterprises, Bellman's optimality principle

Language: Russian

Citation: Galiaskarova GR, Kulinich OV (2014) DYNAMIC MODEL OF OPTIMAL PLACEMENT OF INDUSTRIAL ENTERPRISES. ISJ Theoretical \& Applied Science 10 (18): 25-27. doi: http://dx.doi.org/10.15863/TAS.2014.10.18.6

\section{ДИНАМИЧЕСКАЯ МОДЕЛЬ ОПТИМАЛЬНОГО РАСПРЕДЕЛЕНИЯ ПРОИЗВОДСТВЕННЫХ ПРЕДПРИЯТИЙ}

Аннотация: Была построена динамическая модель оптимального размещения хлебобулочных предприятий в нескольких районах города. Данная модель позволяет рационально распределить строительство новых предприятий в нескольких районах города, либо городах путем сокращения затрат на их строительство.

Ключевые слова: динамическая программирование, оптимальное размещуение предприятий, принщип оптимальности Беллмана.

Динамическое программирование - один из наиболее мощных методов оптимизации. Данный метод применяется для решения многих экономических задач. К примеру, методом динамического программирования решаются такие задачи как:

1) оптимальная стратегия замены оборудования;

2) оптимальное распределение ресурсов;

3) распределение инвестиций для эффективного использования потенциала предприятия;

4) минимизация затрат на строительство и эксплуатацию предприятий;

5) нахождение рациональных затрат при строительстве трубопроводов и транспортных артерий.

С задачами принятия рациональных решений, выбора наилучших вариантов, оптимального управления имеют дело специалисты разного профиля. Среди методов оптимизации динамическое программирование занимает особое положение. Этот метод исключительно привлекателен благодаря простоте и ясности своего основного принципа - принципа оптимальности. Сфера приложения принципа оптимальности чрезвычайно широка, круг задач, к которым он может быть применен, до настоящего времени еще полностью не очерчен [2]. Но сама суть принципа оптимальности заключается в том, что каково бы ни было состояние системы в результате какого-либо числа шагов, на ближайшем шаге нужно выбирать управление так, чтобы оно в совокупности с оптимальным управлением на всех последующих шагах приводило к оптимальному выигрышу на всех оставшихся шагах, включая данный. На каждом шагу ищется такое управление, которое обеспечивает оптимальное продолжение процесса относительно достигнутого в данный момент состояния. Процесс управления должен быть без обратной связи, т.е. управление на данном шаге 
не должно оказывать влияния на предшествующие шаги [4].

Потребительский спрос является ведущим фактором экономического роста индустриально развитых стран [5]. Поэтому важной задачей любого государства является полное удовлетворение спроса населения продукцией, предлагаемой предприятиями.

Часто случается, что наличных мощностей предприятий по производству продукции недостаточно для удовлетворения спроса потребителей. Это требует ввода новых мощностей за счет капитального строительства или реконструкции. Обычно существует несколько вариантов строительства и реконструкции, отличающихся по производственной мощности, местоположению, уровню инвестиционных вложений и другим показателям. И перед производителем встает задача рационального распределения производств. В этом случае прибегают к задаче оптимального размещения производственных предприятий.

Теория оптимального распределения ресурсов и размещения производств - это теория, которая предусматривает формулирование статистической и динамической моделей текущего и перспективного планирования использования ресурсов на базе новых математических подходов в сфере системного построения экономических показателей, используемых для анализа ценообразования, эффективности капитальных вложений. [1]

Экономико - математическая модель задачи позволяет одновременно решать задачу оптимального закрепления потребителей к поставщикам и задачу выбора оптимального варианта размещения производства [6].

Рассматривалась задача планирования предпринимателем строительства пяти предприятий одинаковой мощности по выпуску хлебобулочных изделий, пользующихся спросом, в трех районах города.

Таблица 1

Затраты на строительство g-предприятий в $x_{i}$ районе

\begin{tabular}{|l|l|l|l|l|l|l|}
\hline xi $\backslash$ g & 0 & 1 & 2 & 3 & 4 & 5 \\
\hline g1 & 0 & 11 & 18 & 35 & 51 & 76 \\
\hline g2 & 0 & 10 & 19 & 34 & 53 & 75 \\
\hline g3 & 0 & 9 & 20 & 36 & 54 & 74 \\
\hline
\end{tabular}

Целью планирования явилось размещение предприятий таким образом, чтобы обеспечить минимальные суммарные затраты на их строительство и эксплуатацию, т.е. минимизировать общую величину затрат при освоении ресурса $x$ всеми способами:

$$
\varphi_{n}(x)=\min \sum_{i=1}^{n} g_{i}\left(x_{i}\right)
$$

при ограничениях:

$$
\begin{aligned}
\sum_{i=1}^{n} x_{i} & =x, \\
x_{i} & \geq 0 \mathrm{i}=\overline{1, n} .
\end{aligned}
$$

Решение задачи проводилось с использованием рекуррентных соотношений. Так для первого района:

$$
\varphi_{1}(x)=\operatorname{ming}_{i}\left(x_{i}\right)=g_{1}(x),
$$

для остальных районов:

$$
\begin{gathered}
\varphi_{k}(x)=\min \left\{g_{i}\left(x_{k}\right)+\varphi_{k-1}\left(x-x_{k}\right)\right\}, \\
k=\overline{2, n},
\end{gathered}
$$

где $x$ - количество распределяемого ресурса, которое можно использовать $\mathrm{n}$ различными способами;

$x_{i}$ - количество ресурса, используемого по іму способу $(\mathrm{i}=\overline{1, n})$;

$g_{i}\left(x_{i}\right)$ - функция расходов, равная величине затрат на производство при использовании pecурса $x_{i}$ по і-му способу;

$\varphi_{k}(x)$ - наименьшие затраты, которые нужно произвести при использовании ресурса $x$ первыми $k$ способами.

Задача решалась в 3 этапа:

на первом этапе были найдены затраты, равные 76 млн. руб., которые могли быть получены при строительстве всех пяти предприятий в одном районе;

на втором этапе была определена оптимальная стратегия размещения предприятий только в двух первых районах, которая оказалась равной 52 млн. руб.;

на заключительном этапе определялась оптимальная стратегия размещения пяти предприятий в трех районах, составившая 46 млн. руб.

Аналогичный результат был получен используя принцип оптимальности Беллмана. Расчеты проводились в MS Excel.

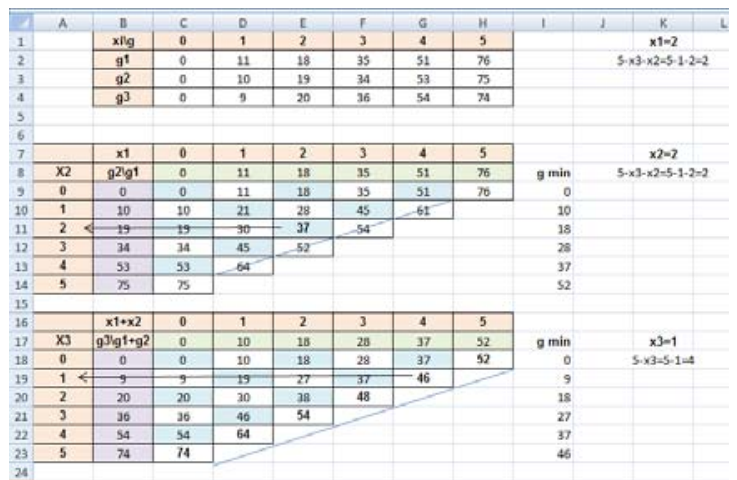

\section{Рисунок 1 - Расчеты рационального размещения предприятий по принципу оптимальности Беллмана}

Таким образом, решив данную задачу, можно сделать вывод, что оптимальная стратегия состоит в строительстве одного предприятия в третьем районе, по два предприятия во втором и первом 
районах, при этом минимальная стоимость строительства и эксплуатации составит 46 млн. руб.

Метод динамического программирования один из наиболее мощных и широко известных математических методов современной теории управления, был предложен в конце 50-х годов американским математиком P. Беллманом и быстро получил широкое распространение, чему способствовали ярко и доходчиво написанные книги самого Беллмана, которые были быстро переведены на русский язык [9]. Формулировка метода динамического программирования, данная Беллманом, а также многочисленные приложения метода к разнообразным проблемам теории принятия решения, экономики, экологии и других областей знания способствовали закреплению этого метода как одного из важнейших инструментов теории управляемых процессов [10].

Динамическое программирование определяет оптимальное решение n-мерной задачи путем ее декомпозиции на $\mathrm{n}$ этапов, каждый из которых представляет собой подзадачу относительно одной переменной. Вычислительное преимущество такого подхода состоит в том, что занимаются решением одномерных оптимизационных задач подзадач вместо большой n-мерной задачи, а затем собираем решение основной задачи «снизу вверх». Динамическое программирование применимо тогда, когда подзадачи не являются независимыми, иными словами, когда у подзадач есть общие «подподзадачи» [4]. Алгоритм, основанный на динамическом программировании, решает каждую из подзадач единожды и запоминает ответы в специальной таблице. Это позволяет не вычислять заново ответ к уже встречавшейся подзадаче.

В типичном случае динамическое программирование применяется к задачам оптимизации. У такой задачи может быть много возможных решений; их «качество» определяется значением какого-то параметра, и требуется выбрать оптимальное решение, при котором значение параметра будет минимальным или максимальным (в зависимости от постановки задачи). Вообще говоря, оптимум может достигаться для нескольких разных решений [8].

Автор динамического программирования $\mathrm{P}$. Беллман сформулировал принцип оптимальности: каково бы ни было начальное состояние на любом шаге и решение, выбранное на этом шаге, последующие решения должны выбираться оптимальными относительно состояния, к которому придет система в конце данного шага [7]. Использование этого принципа гарантирует, что решение, выбранное на любом шаге, является не локально лучшим, а лучшим с точки зрения задачи в целом.

Данный метод уже получил свое распространения и, без сомнения, будет иметь успех и в будущем, так как обладает замечательной особенностью - способностью усовершенствовать решение задач, решаемых, например, с помощью рекурсий или перебора вариантов.

\section{References:}

1. (2011) Modern economic theory in the hands of nobeliatov. Ed. Academy of Natural History, 218.

2. (2014) Economic-mathematical methods: electronic textbook.

3. Howard R (1964) Dynamic programming and Markov processes. - Moscow: Sov. radio, 462.

4. Bellman R (1960) Dynamic Programming. Moscow: Izd. Lita., 400.

5. Ostrovsky IE (2012) Mathematical modeling of industrial and economic processes and systems. Comp.: Maritime GSKHA.- Ussuriysk, 35.

6. Krass MS, Chupryna BP (2003) The foundations of mathematics and its applications in economic education, 4th ed., Rev. - Moscow: Business, 688 .
7. (2014) Electronic resource: Dynamic programming and greedy. Available: http://kitnet.altnet.ru/www/metod/book3/doc1/s tr1.htm

8. (2014) Electronic resource: Dynamic programming Available: http://www.edu.nstu.ru/courses/saod/alg_dinam htm

9. (2014) Electronic resource: Available: http://www.uecs.ru/uecs51-512013/item/20292013-03-13-08-02-15

10. (2014) Electronic resource: Available: http://masters.donntu.edu.ua/2002/kita/serdyuk/ diss/lib/3/index $3 . h t m$ 